\title{
Acetyl-l-carnitine enhances myelination of regenerated fibers of the lateral olfactory tract
}

Nanae Fukushima ${ }^{\mathrm{a}, *}$, Kumiko Yokouchi ${ }^{\mathrm{a}}$, Masafumi Kuroiwa ${ }^{\mathrm{b}}$, Kyutaro Kawagishi ${ }^{\mathrm{a}}$, Tetsuji Moriizumi ${ }^{\mathrm{a}}$

${ }^{a}$ Department of Anatomy, Shinshu University School of Medicine, Matsumoto, Nagano 390-8621, Japan

${ }^{b}$ Department of Neurosurgery, Shinshu University School of Medicine, Matsumoto, Nagano 390-8621, Japan

*Corresponding author at: Department of Anatomy, Shinshu University School of Medicine, 3-1-1 Asahi, Matsumoto, Nagano 390-8621, Japan.

E-mail address: nanae@shinshu-u.ac.jp (N. Fukushima).

Abbreviations: ALC, acetyl-1-carnitine; FB, fast blue; LOT, lateral olfactory tract; MBP, myelin basic protein; $\mathrm{P}$, postnatal day; $\mathrm{SD}$, standard deviation. 


\begin{abstract}
It is well known that acetyl-1-carnitine (ALC) has various neuroprotective effects against neurodegenerative diseases. In addition, it has been reported that ALC facilitates myelination of regenerated axons after peripheral nerve injuries. We previously reported that spontaneous regeneration of the lateral olfactory tract (LOT), the main fiber tract of the central olfactory system, consistently occurred in newborn rats and a majority of these regenerated fibers were unmyelinated in neonatally LOT-transected young adult rats. To investigate the effects of ALC treatment on myelination in LOT, neonatal rats were treated with ALC after LOT transection. Immunohistochemistry for myelin basic protein showed more positive areas in ALC-treated rats than in control rats. Moreover, the number of myelinated axons of regenerated fibers was assessed using electron microscopy and was found to be statistically higher in ALC-treated rats compared to control rats. The study revealed that ALC accelerates myelination of regenerated fibers in neonatally LOT-injured young adult rats.
\end{abstract}

Keywords:

Olfactory tract injury

Spontaneous regeneration

Acetyl-1-carnitine

Myelinated axons

Neonatal rats 


\section{Introduction}

L-carnitine or acetyl-1-carnitine (ALC), the acetyl derivative of 1-carnitine, has various neuroprotective effects against neurodegenerative diseases, such as Alzheimer's disease, Parkinson's disease, ischemia, and traumatic brain injury [1-5]. Moreover, it has been reported that 1-carnitine or ALC facilitates myelination of regenerated axons after peripheral nerve injuries [6-8]. In the central nervous system, 1-carnitine or ALC is also reported to enhance myelin formation, but this finding is limited to a few studies [9, $10]$.

The lateral olfactory tract (LOT) is the main fiber tract of the central olfactory system and exclusively consists of myelinated axon fibers. We have recently demonstrated that the neonatally transected LOT can be replaced by newly-formed axon fibers connecting the olfactory bulb and the olfactory cortex (the olfactory tubercle and piriform cortex) via the transected site at a later stage [11-14]. Because the regenerated LOT is primarily composed of unmyelinated axon fibers, LOT injury is a good animal model to examine the effects of ALC on myelin formation in the developing brain. Therefore, we used neonatal rats in our injury model of the central olfactory tract to investigate whether ALC accelerates myelination and increases the number of myelinated axon fibers in the regenerated LOT of young adult rats.

\section{Materials and methods}

\subsection{Animals}

Newborn Wistar rat pups (Japan SLC Inc., Hamamatsu, Japan) of both sexes were used for this study. Postnatal day (P) 0 refers to the first $24 \mathrm{~h}$ after birth. All procedures were conducted in accordance with the National Institutes of Health Guide for the Care 
and Use of Laboratory Animals, and protocols were approved by our Institutional Animal Care and Use Committee. All efforts were made to minimize the number of animals used and their suffering. Surgical manipulations were performed in hypothermic conditions using a freezer $\left(-20^{\circ} \mathrm{C}, 15-20 \mathrm{~min}\right)$.

\subsection{LOT transection and retrograde tracer injection}

LOT transection was performed in P2 pups $(n=70)$ unilaterally on the left side, as described previously [11-13]. Briefly, the LOT was transected at the posterior half of the olfactory stria by inserting the tip of a knife (Ophthalmic Scleral MVR Knife, 25 gauge; Alcon, Tokyo, Japan) from the ventrolateral aspect of the head. Immediately after LOT transection, a retrograde fluorescent tracer, Fast Blue (FB) (Polysciences Inc., Warrington, PA, USA), was injected into the left olfactory cortex to confirm the completeness of LOT transection. FB $(1 \%, 0.1 \mu \mathrm{l})$ was injected into the posterior part of the olfactory cortex situated far caudal to the site of LOT transection. After surgery, the pups were housed with their dam in a single cage $(26.0 \times 42.0 \times 18.0 \mathrm{~cm})$ under standard laboratory conditions with a 12-h light/dark cycle and room temperature of $22^{\circ} \mathrm{C}$. Food and water were supplied ad libitum. In total, 14 pups that underwent LOT transection on P2 showed a continual decrease in body weight and died by P5.

\subsection{ALC injection and tissue preparation}

The LOT-transected rat pups were randomly divided into two groups, based on sex and weight, ALC-treated $(n=28)$ and control $(n=28)$ groups. Each rat in the ALC-treated group was injected intraperitoneally once daily from P5 to P20 with 100 $\mathrm{mg} / \mathrm{kg}$ body weight of ALC (Sigma-Aldrich, Saint Louis, MO, USA). The rats in the 
control group were injected with an equivalent volume of saline. On P30, rats were euthanized with sodium pentobarbital (100 mg/kg, intraperitoneally) and perfused through the heart with $50 \mathrm{~mL}$ of $4 \%$ paraformaldehyde in $0.1 \mathrm{M}$ phosphate buffer. The brains were removed, postfixed for 2 days in the same fixative, soaked in $30 \%$ sucrose for 2 days, and divided into 2 regions (olfactory bulbs and other brain regions). Using a freezing microtome, $50 \mu \mathrm{m}$ sagittal sections of the olfactory bulb were cut at $150 \mu \mathrm{m}$ intervals ( 3 sets). Other brain regions were cut into a series of $50 \mu \mathrm{m}$ coronal sections at $300 \mu \mathrm{m}$ intervals (6 sets). The first set of sections was mounted as a series on coated slides, cover-slipped with glycerol, and observed under a fluorescence microscope to exclude incomplete LOT-transected cases with FB-positive bulbar mitral cells.

The second set of coronal brain sections from the ALC-treated and control groups with complete LOT transection was immunostained using an anti-myelin basic protein (MBP) antibody to detect the myelin sheaths. The sections were immersed in $0.3 \%$ $\mathrm{H}_{2} \mathrm{O}_{2}$ for $30 \mathrm{~min}$ to suppress endogenous peroxidase activity and for $2 \mathrm{~h}$ in phosphate-buffered saline containing $0.3 \%$ Triton $\mathrm{X}-100$. They were then incubated overnight with mouse anti-MBP antibody $(1: 10,000$; Protos Biotech Corporation, New York, NY, USA). After washing, the sections were incubated with biotinylated anti-mouse immunoglobulin (1:500; Dako, Glostrup, Denmark) for $2 \mathrm{~h}$ and then with streptavidin-peroxidase (1:500; Dako) for $2 \mathrm{~h}$. The peroxidase reaction product was visualized using the Metal Enhanced DAB Substrate Kit (Thermo Fisher Scientific Inc., Pierce Biotechnology, Rockford, IL, USA) (diaminobenzidine, 0.01\%; $\mathrm{H}_{2} \mathrm{O}_{2}, 0.0015 \%$ ). The sections were rinsed, mounted on coated slides, air-dried, dehydrated, and cover-slipped with Entellan New (Merck, Darmstadt, Germany). 


\subsection{Measurement of MBP-positive areas}

In the coronal sections of MBP staining at the level of the middle of the olfactory tubercle from the ALC-treated and control groups with complete LOT transection, the MBP-positive areas on the left transected and right uninjured sides were quantified using the Image J $1.48 \mathrm{v}$ software. The data were expressed as the mean \pm standard deviation of the mean (SD). Statistical differences between the means were evaluated using Student's $t$-test. $P$ values $<0.05$ were considered to be statistically significant.

\subsection{Quantification of myelinated axons in the LOT}

For each sample from both the ALC-treated and control groups with complete LOT transection, one section was selected from the adjacent coronal brain sections of MBP staining at the level of the middle of olfactory tubercle. Small pieces of tissue containing the LOT from both sides were dissected out and processed for electron microscopy. They were postfixed with buffered $2 \%$ osmium tetroxide, dehydrated in ethanol, processed with propylene oxide, and embedded in Epon. Ultrathin sections were briefly stained with lead citrate and observed with an electron microscope (JEM-1400; JEOL Ltd., Tokyo, Japan). In each section, 10 randomly selected fields $(12.5 \times 19.0 \mu \mathrm{m})$ containing myelinated axon fibers were captured and the numbers of myelinated axons were counted. The data were expressed as the mean $\pm \mathrm{SD}$. Statistical differences between two data sets were evaluated using Student's $t$-test. $P$ values $<0.05$ were considered to be statistically significant.

\section{Results}

\subsection{Confirmation of the completeness of LOT transection}


Several rats in the ALC-treated $(n=5)$ and control $(n=5)$ groups died during the experiment due to neglect or abandonment by the mother. Ultimately, brain sections from the ALC-treated $(n=23)$ and control $(n=23)$ groups were observed under a fluorescence microscope. Fig. 1A shows a representative case of a P30 rat that underwent unilateral transection of the left LOT on P2. Histological examination revealed that 12 ALC-treated rats and 18 control rats with neonatal LOT transection contained varying numbers of FB-positive mitral cells in the left olfactory bulb (Fig. 1B and C) and were regarded as incomplete LOT-transected cases. In the complete LOT-transected cases (ALC-treated, $n=11$; control, $n=5$ ), there were no FB-positive mitral cells in the left olfactory bulb (Fig. 1D).

\subsection{The effect of ALC treatment on myelination of regenerating axons}

Immunohistochemical staining for MBP showed a near-absence of MBP-positive fibers on the left LOT-transected side in the control rat with complete LOT transection (Fig. 2A). Meanwhile, in the ALC-treated rat with complete LOT transection (Fig. 2B), thin MBP-positive myelinated components were present in the outer superficial layers of the olfactory tubercle and piriform cortex on the left transected side. The thicknesses of the MBP-positive areas on the left transected side in the ALC-treated rats $(0.84 \pm$ $\left.0.22 \times 10^{5} \mu \mathrm{m}^{2}\right)$ were much greater than those in the control rats $\left(0.25 \pm 0.14 \times 10^{5}\right.$ $\mu \mathrm{m}^{2}$ ) and a statistically significant difference was found between the control and ALC-treated rats $(p<0.01)$. On the right uninjured side, MBP-positive areas were similar both in the control $\left(1.39 \pm 0.19 \times 10^{5} \mu \mathrm{m}^{2}\right)$ and ALC-treated rats $(1.44 \pm 0.20 \times$ $10^{5} \mu \mathrm{m}^{2}$ ) and there was no statistical significance between the two groups. 
Fig. 3 shows electron microscopic images of the LOT in the control (Fig. 3A and B) and ALC-treated (Fig. 3C and D) rats with complete LOT transection. On the left transected side, there were more myelinated axons in the ALC-treated rats than in the control rats. The average number of myelinated axon fibers per microscopic field image on the left transected side in the control and ALC-treated rats was $5.5 \pm 2.2$ and $35.6 \pm$ 8.2, respectively, and a statistically significant difference was found between the control and ALC-treated rats (Fig. 4). On the other hand, in the both groups, there were many axons with myelin sheaths on the right uninjured side and the average number of myelinated axon fibers was $69.8 \pm 5.9$ and $70.9 \pm 5.2$ in the control and ALC-treated rats, respectively. There was no statistical significance between the two groups for the uninjured right side (Fig. 4).

\section{Discussion}

Several studies have shown that 1-carnitine or ALC has a protective effect on axon and myelin sheath degeneration after peripheral nerve injuries $[6-8,15]$. Moreover, Ueno et al. [10] reported that l-carnitine suppresses oxidative stress in oligodendrocytes after ligation of the bilateral common carotid arteries and enhances myelin sheath growth and facilitates myelination of axons in the white matter lesions of the brain after hypoperfusion. In our LOT injury model with its complete transection, LOT regions at the level of the olfactory tubercle, that is, located far caudal to the transected site of LOT, are regarded to be entirely composed of regenerated axon fibers. Because, as mentioned earlier, those regenerated axon fibers are mostly unmyelinated, analysis of the regenerated LOT allows reliable quantification of the effect of ALC on myelin formation around regenerated fibers. In the neonatal rats, the axons in the LOT are still 
unmyelinated at the time of transection (P2) and at the time of starting ALC treatment (P5), and thus we consider that increased myelinated axons by ALC treatment are not due to its protective effect on myelin sheath but its facilitating effect on myelin formation. The present study showed that the number of myelinated axons greatly increased approximately 6.5 -fold by ALC treatment. This value is much higher than the previously reported value of a 1.4-fold increase by Wilson et al. [7] and 2.1-fold increase by Farahpour and Ghayour [8], although it should be noted that these data cannot be directly compared because of differences between central and peripheral nervous systems.

ALC was administered daily between P5 and P20. After LOT transection on P2, axonal regeneration proceeds in the olfactory tubercle between P7 and P12 with the fastest components of regenerating axon fibers having an approximate elongation speed of $0.4 \mathrm{~mm}$ per day [14]. It remains to be determined whether critical periods regarding the effectiveness of ALC on myelin formation exist during the developmental stages: the early stage during fiber elongation between P5 and P12 and/or the later stage just after fiber elongation between P13 and P20 or the stage long after the completion of LOT regeneration between $\mathrm{P} 30$ and $\mathrm{P} 37$.

We have recently reported regeneration of LOT in developing rats during the early suckling period $[11,13]$, and such regeneration of LOT had critical periods early in life [13]. Interestingly, the regenerated olfactory system was functional 10 days [14] and 4 weeks [11] after LOT transection at the neonatal stage despite the fact that regenerated axon fibers mostly lacked myelin sheaths. In consideration of the finding that ALC treatment resulted in a significant number of myelinated axon fibers in the regenerated 
LOT, it would be of interest to conduct electrophysiological studies on nerve conduction velocity through the regenerated LOT with and without ALC treatment.

\section{Conclusion}

The present results revealed that ALC accelerated the myelination of regenerated fibers in neonatally LOT-injured young adult rats.

\section{Conflicts of interest}

The authors have no conflicts of interest to declare.

\section{Funding}

This work was supported by JSPS KAKENHI (Grant Number JP15K08131). 


\section{References}

[1] J.W. Pettegrew, W.E. Klunk, K. Panchalingam, J.N. Kanfer, R.J. McClure, Clinical and neurochemical effects of acetyl-L-carnitine in Alzheimer's disease, Neurobiol. Aging 16 (1995) 1-4.

[2] S. Scafidi, J. Racz, J. Hazelton, M.C. McKenna, G. Fiskum, Neuroprotection by acetyl-L-carnitine after traumatic injury to the immature rat brain, Dev. Neurosci. $32(2010) 480-487$.

[3] S.A. Zaitone, D.M. Abo-Elmatty, A.A. Shaalan, Acetyl-L-carnitine and $\alpha$-lipoic acid affect rotenone-induced damage in nigral dopaminergic neurons of rat brain, implication for Parkinson's disease therapy, Pharmacol. Biochem. Behav. 100 (2012) 347-360.

[4] R.F. Villa, F. Ferrari, A. Gorini, ATP-ases of synaptic plasma membranes in striatum: enzymatic systems for synapses functionality by in vivo administration of L-acetylcarnitine in relation to Parkinson's Disease, Neuroscience 248 (2013) 414 426.

[5] K. Kocsis, L. Knapp, L. Gellert, G. Olah, Z. Kis, H. Takakuwa, N. Iwamori, E. Ono, J. Toldi, T. Farkas, Acetyl-L-carnitine normalizes the impaired long-term potentiation and spine density in a rat model of global ischemia, Neuroscience 269 (2014) 265-272.

[6] C. De Angelis, C. Scarfò, M. Falcinelli, E. Perna, E. Reda, M.T. Ramacci, L. Angelucci, Acetyl-L-carnitine prevents age-dependent structural alterations in rat peripheral nerves and promotes regeneration following sciatic nerve injury in young and senescent rats, Exp. Neurol. 128 (1994) 103-114. 
[7] A.D. Wilson, A. Hart, M. Wiberg, G. Terenghi, Acetyl-1-carnitine increases nerve regeneration and target organ reinnervation - a morphological study, J. Plast. Reconstr. Aesthet. Surg. 63 (2010) 1186-1195.

[8] M.R. Farahpour, S.J. Ghayour, Effect of in situ delivery of acetyl-L-carnitine on peripheral nerve regeneration and functional recovery in transected sciatic nerve in rat, Int. J. Surg. 12 (2014) 1409-1415.

[9] M.T. Ramacci, M. De Rossi, M.R. Lucreziotti, M.C. Mione, F. Amenta, Effect of long-term treatment with acetyl-L-carnitine on structural changes of ageing rat brain, Drugs Exp. Clin. Res. 14 (1988) 593-601.

[10]Y. Ueno, M. Koike, Y. Shimada, H. Shimura, K. Hira, R. Tanaka, Y. Uchiyama, N. Hattori, T. Urabe, L-carnitine enhances axonal plasticity and improves white-matter lesions after chronic hypoperfusion in rat brain, J. Cereb. Blood Flow Metab. 35 (2015) 382-391.

[11]M. Sakamoto, K. Yokouchi, Y. Sekiguchi, N. Fukushima, K. Kawagishi, A. Kakegawa, N. Sumitomo, T. Moriizumi, Re-evaluation of spontaneous regeneration of the lateral olfactory tract, Neurosci. Res. 68 (2010) 15-21.

[12]N. Fukushima, K. Yokouchi, M. Sakamoto, Y. Sekiguchi, H. Koike, K. Kawagishi, T. Moriizumi, Analysis of spontaneous regeneration of olfactory structures with emphasis on myelination and re-innervation of cortical areas, Neurosci. Lett. 537 (2013) 35-39.

[13]S. Hirayama, K. Kawagishi, K. Yokouchi, N. Fukushima, M. Karasawa, T. Moriizumi, Regenerative capacity of bulbar projection neurons during development: a quantitative neuronal analysis with functional correlation, Chem. Senses 39 (2014) 47-56. 
[14]M. Kuroiwa, N. Fukushima, K. Yokouchi, K. Kawagishi, T. Moriizumi, Morphological analysis of regenerated bulbar fibers in relation to neonatal olfaction, Brain Res. Bull. 127 (2016) 66-73.

[15]U.Z. Avsar, U. Avsar, A. Aydin, M. Yayla, B. Ozturkkaragoz, H. Un, M. Saritemur, T. Mercantepe, L-carnitine alleviates sciatic nerve crush injury in rats: functional and electron microscopy assessments, Neural Regen. Res. 9 (2014) 1020-1024. 


\section{Figure legends}

Fig. 1. The image shows the ventral view of a completely LOT-transected case (A). An arrow points to the transected site and the left transected LOT cannot be seen as a white myelinated band due to lack of myelination. LOT, lateral olfactory tract; OB, olfactory bulb; OT, olfactory tubercle. The images (B-D) show the bulbar mitral cell layers of incompletely LOT-transected cases (B and C) and a completely LOT-transected case (D). FB-positive mitral cells can be seen in the left olfactory bulb of incompletely LOT-transected cases (B and C), but they are absent in the completely LOT-transected case (D). Scale bar, B-D, $100 \mu \mathrm{m}$.

Fig. 2. The images show the sections processed for immunohistochemistry using an anti-MBP antibody from the control (A) and ALC-treated (B) rats. Upper, middle, and lower images are from the level of the rostral one-third, middle, and caudal one-third of the olfactory tubercle, respectively. Note the increase in MBP-positive myelinated fibers (arrows) on the left LOT-transected side in the ALC-treated rat. Scale bars, $1 \mathrm{~mm}$.

Fig. 3. The images show the electron microscopic pictures of the LOT from the adjacent sections of middle images of Figure 2 in the control (A and B) and ALC-treated (C and D) rats on the right uninjured (A and C) and left LOT-transected (B and D) sides. Note the increase in myelinated axon fibers on the left transected side in the ALC-treated rat. Scale bars, $2 \mu \mathrm{m}$.

Fig. 4. The graph shows the number of myelinated axons on the right uninjured and left LOT-transected sides in the control and ALC-treated rats. Data are presented as the 
mean \pm SD. ${ }^{* *} p<0.01$ 
Fig. 1

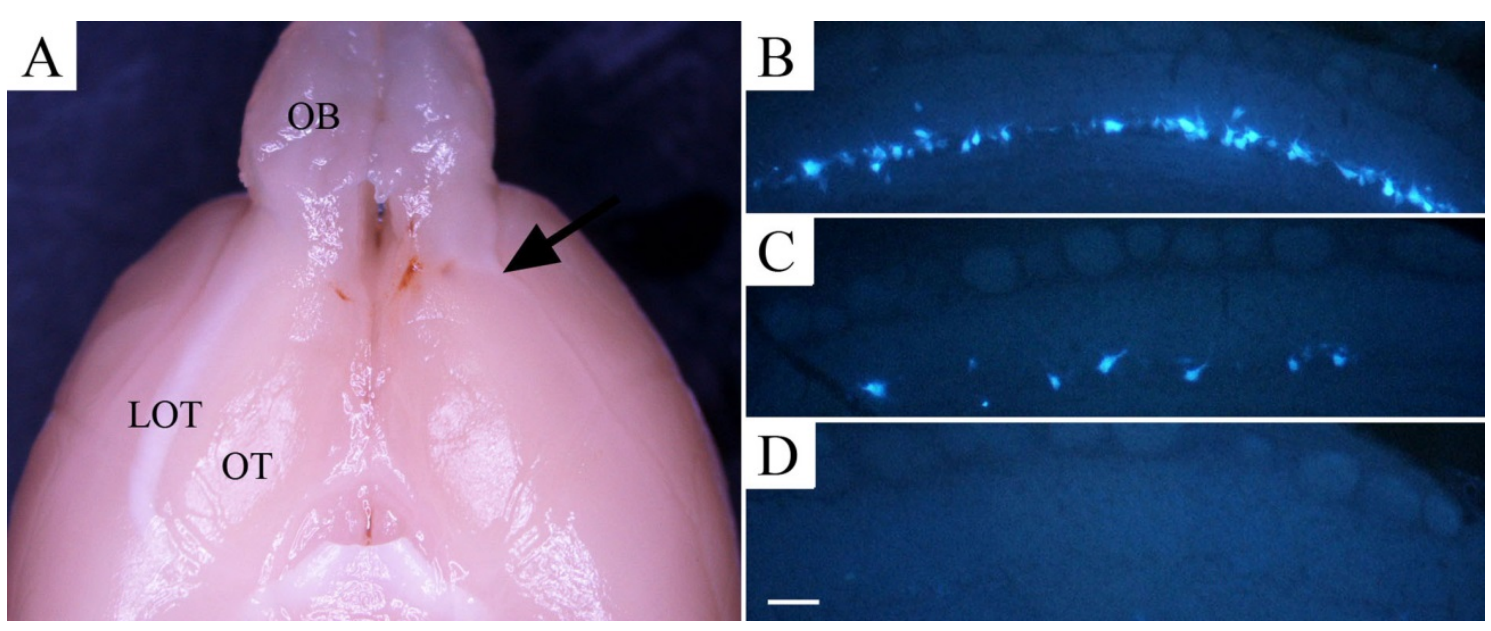

Fig. 2

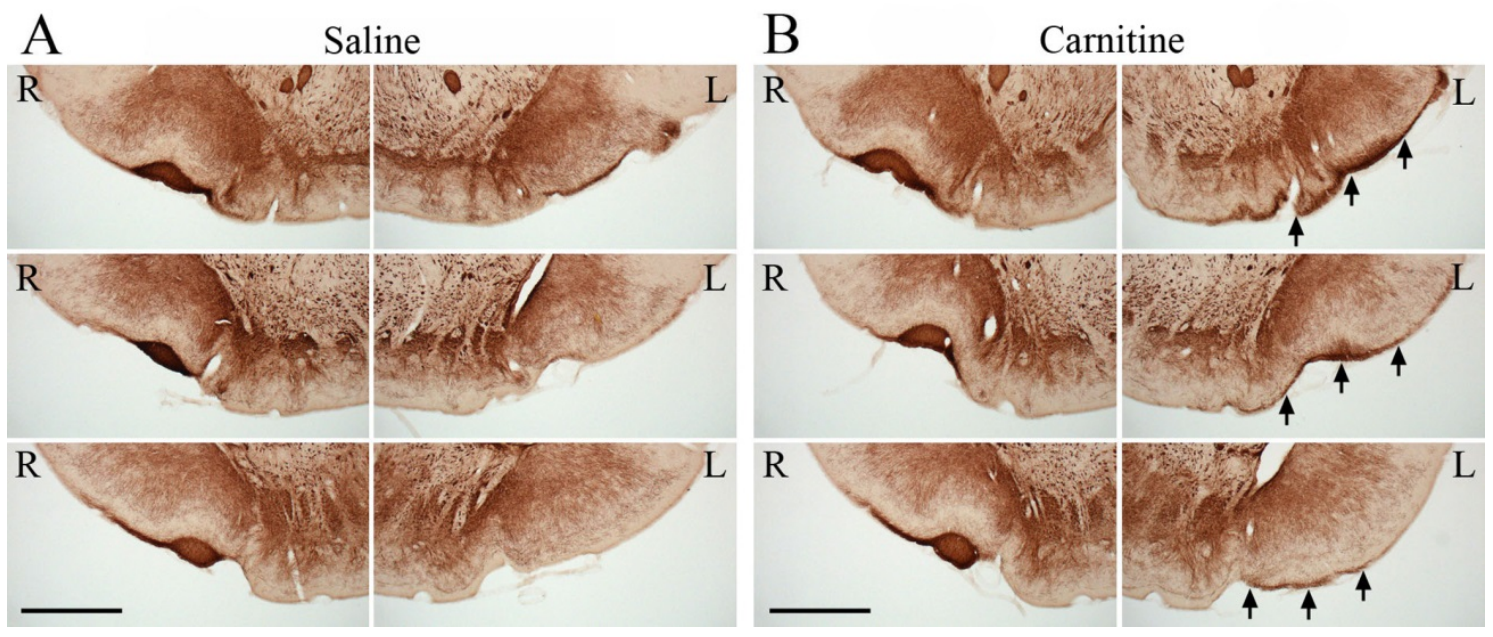


Fig. 3
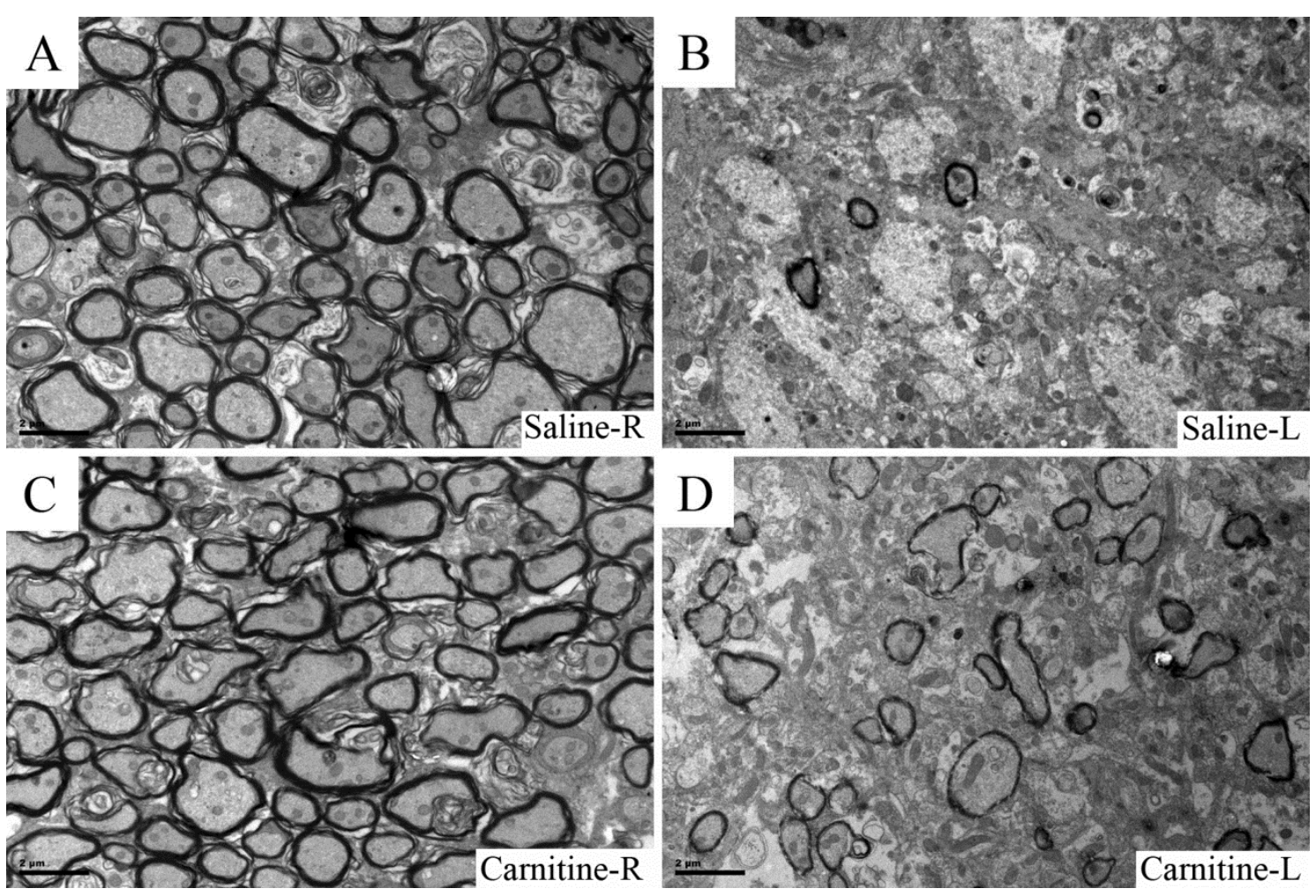

Fig. 4

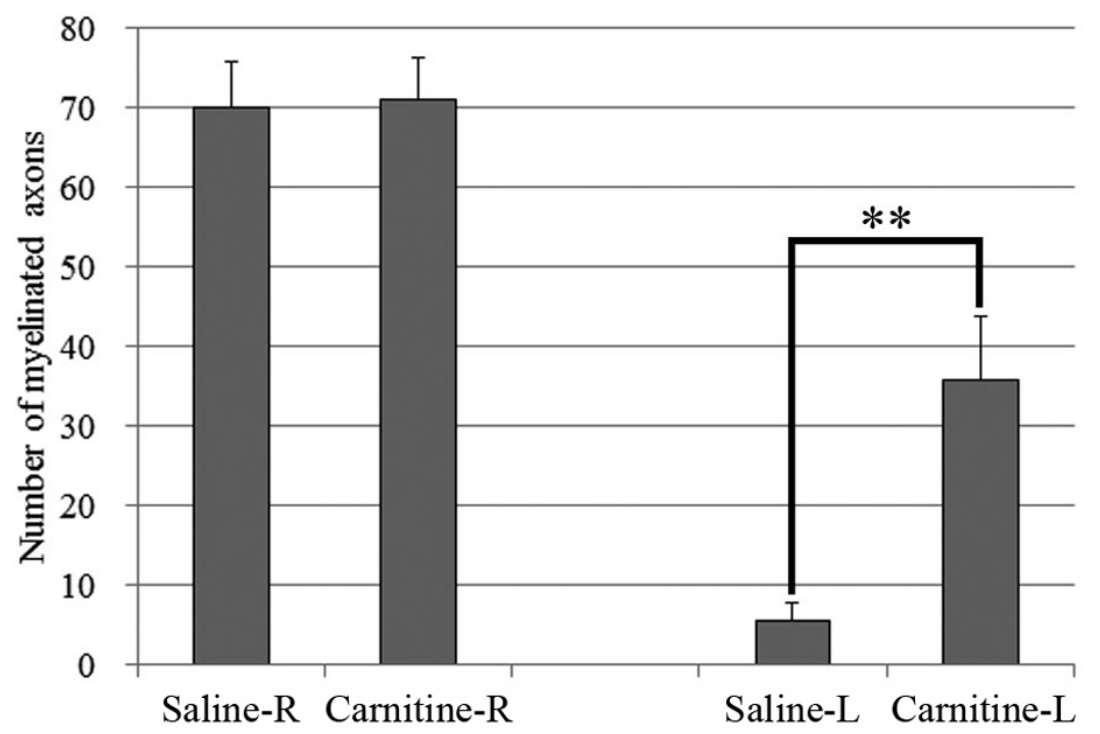

